\title{
Host immune response to Chlamydia pneumoniae heat shock protein 60 is associated with asthma
}

\author{
T. Huittinen*, D. Hahn", T. Anttila*, E. Wahlström ", P. Saikku*,+, M. Leinonen*
}

Host immune response to Chlamydia pneumoniae heat shock protein 60 is associated with asthma. T. Huittinen, D. Hahn, T. Anttila, E. Wahlström, P. Saikku, M. Leinonen. (C) ERS Journals Ltd 2001.

ABSTRACT: Chlamydia pneumoniae infection has been associated with asthma. It has also been suggested that heat shock protein 60 (Hsp60) belonging to a class of highly conserved proteins may play a role in the pathogenesis of chlamydial infections. The purpose was to study whether the host immune response to $C$. pneumoniae Hsp60 is associated with asthma and decreased pulmonary function.

An enzyme immunoassay was used to measure immunoglobulin-(Ig)A and IgG antibodies against recombinant $C$. pneumoniae Hsp60 and human $\mathrm{Hsp60}$ in a study group consisting of 24 cases of recently symptomatic asthma and 62 nonasthmatic controls.

A strong $(\mathrm{r}=0.50)$ and significant $(\mathrm{p}<0.001)$ correlation was observed between $C$. pneumoniae and human $\mathrm{Hsp60} \mathrm{IgA}$ antibodies, but only $C$. pneumoniae $\mathrm{Hsp60} \mathrm{IgA}$ antibodies were significantly associated with asthma $(p=0.02)$. Pulmonary function, as measured by forced expiratory volume in one second, also inversely correlated $(\mathrm{r}=\mathbf{- 0 . 2 3}, \mathrm{p}=\mathbf{0 . 0 4})$ with the quantity of $C$. pneumoniae Hsp60 IgA antibodies, suggesting an association with the severity of pulmonary obstruction.

By showing an association of Chlamydia pneumoniae heat shock protein 60 immunoglobulin $\mathrm{A}$ antibodies with asthma, the results support the hypothesis of an association between Chlamydia pneumoniae infection and asthma and support the need for further investigations on the role of heat shock protein 60 in the pathogenesis of asthma.

Eur Respir J 2001; 17: 1078-1082.
* National Public Health Institute, Oulu, Finland, " Dean Medical Center, Madison, Wisconsin, USA, National Public Health Institute, Helsinki, Finland. ${ }^{+}$Dept of Medical Microbiology, University of Oulu, Oulu, Finland

Correspondence: T. Huittinen, National Public Health Institute, P.O. Box 310, FIN-90101 Oulu, Finland.

Fax: 35885376251

Keywords: Asthma

Chlamydia pneumoniae

heat shock protein

serology

Received: October 122000

Accepted after revision January 25 2001

This study was supported by a grant from the Sigrid Juselius Foundation and the contract BIO4-CT96-0152 of the Biotechnology Programme of the Commission of the European Union.
A growing body of evidence implicates persistent lung infection caused by Chlamydia pneumoniae in the initiation, exacerbation and promotion of asthma symptoms in some affected individuals [1]. A causal association between $C$. pneumoniae infection and asthma is biologically plausible, based on the observations that asthma is a chronic inflammatory disease of the airways and that Chlamydia species are known to produce chronic inflammatory damage in target organs. Whether $C$. pneumoniae lung infections activate the same immunopathological mechanisms that have been demonstrated for other chlamydial diseases has not been explored systematically.

The involvement of the heat shock protein 60 (Hsp60) of C. trachomatis in the immunopathogenesis of trachoma [2], pelvic inflammatory disease [3], tubal infertility [4] and ectopic pregnancy [5] is well known. It is also known that persistent $C$. trachomatis infection in cell culture leads to increased expression of Hsp60 proteins in chlamydia particles [6]. The role of Hsp60 in C. pneumoniae infections has not, however, been studied in adequate detail. Thus, whether the host immune response to the $C$. pneumoniae Hsp60 is associated with asthma and decreased pulmonary function in patients with recently symptomatic asthma was studied.

\section{Material and methods}

\section{Subjects}

Altogether, 86 serum samples from 24 asthma patients and 62 nonasthmatic controls (45 asymptomatic and 17 with acute bronchitis) from a controlled cross-sectional study of adults with recently symptomatic asthma [7], were available in this study. Study subjects were sequentially enrolled during the course of usual care in a private family practice office. Twenty-five asthma patients meeting American Thoracic Society criteria for asthma (appropriate symptoms and forced expiratory volume in one second (FEV1) reversibility) were enrolled in the original study; sera were available from 24 of them for the current study. All asthma patients were ambulatory nonhospitalized cases. Two separate nonasthmatic control groups were enrolled from the same site and time frame: acute bronchitis patients were nonasthmatic adults who had an acute cough syndrome without abnormality in FEV1. Asymptomatic patients were seen for a variety of nonrespiratory reasons (mostly health maintenance examinations). The demographic data of patients and controls are presented in table 1 . 
Table 1. - Characteristics of asthmatic subjects and controls

\begin{tabular}{lccc}
\hline Characteristic & Asthmatic subjects & Asymptomatic controls & Bronchitis controls \\
\hline Subjects n & 24 & 45 & 17 \\
Age yr & $46 \pm 9(37-70)$ & $46 \pm 11(27-80)$ & $46 \pm 10(34-70)$ \\
Sex male & $16(67)$ & $30(67)$ & $9(53)$ \\
Smoking & $6(25)$ & $25(55.6)$ & $6(35.3)$ \\
$\quad$ Never & $9(37.5)$ & $16(35.6)$ & $8(47.1)$ \\
Past & $9(37.5)$ & $4(8.9)$ & $3(17.6)$ \\
Current & $69.2 \pm 13.9$ & $102.0 \pm 8.5$ & $103.7 \pm 12.4$ \\
Prebronchodilator FEV1 \% pred & & & \\
\hline
\end{tabular}

Data presented as mean \pm SD or $\mathrm{n}(\%)$. Due to some missing data average number of packs per year or time since stopping smoking could not be calculated for smoking groups. FEV1: forced expiratory volume in one second.

\section{Heat shock protein 60 serology}

An enzyme immunoassay (EIA) method for the measurement of immunoglobulin-(Ig)A and IgG antibodies against $C$. pneumoniae and human Hsp60 proteins was developed. Immunoplates (Maxisorp F96, Nunc, Roskilde, Denmark) were coated separately with two recombinant Hsp60 proteins: C. pneumoniae Hsp60 produced in Bacillus subtilis and human Hsp60 produced in Escherichia coli (Sigma, St. Louis, MO, USA), both at a concentration of $5 \mu \mathrm{g} \cdot \mathrm{mL}^{-1}$, in $\mathrm{Ca}^{2+}$ and $\mathrm{Mg}^{2+}$ free phosphatebuffered saline (PBS, pH 7.4) overnight at $37^{\circ} \mathrm{C}$. The C. pneumoniae Hsp60 protein was produced by inserting a polymerase chain reaction (PCR) product representing the protein encoding segment of the $C$. pneumoniae GroEL gene extended with six C-terminal "his" codons into a vector for intracellular production in $B$. subtilis, and the protein was purified using metal affinity chromatography (U. Airaksinen, National Public Health Institute, Helsinki, Finland; personal communication). After washing four times with PBS containing $0.05 \%$ Tween 20 (PBS/T) in a plate washer (96 PW, TECAN Austria Ges.m.b.H., Salzburg, Austria), the plates were incubated for $2 \mathrm{~h}$ at $37^{\circ} \mathrm{C}$ with duplicate serum samples diluted $1 / 50$ for human Hsp60 $\operatorname{IgA}$, 1/100 for C. pneumoniae Hsp60 $\operatorname{IgA}$, and $1 / 200$ for both $\mathrm{IgG}$ antibodies in PBS containing $10 \%$ foetal bovine serum (PBS-FBS). The plates were washed as before, and incubated for $2 \mathrm{~h}$ at $37^{\circ} \mathrm{C}$ separately, with alkaline phosphatase-conjugated antihuman IgA (Caltag Laboratories, Burlingame, CA, USA) diluted 1/3,000 in PBS-FBS and antihuman IgG (Sigma) diluted $1 / 1,000$. The plates were first washed three times with PBS/T and then twice with distilled water before incubation at $37^{\circ} \mathrm{C}$ for $30 \mathrm{~min}$ with a substrate solution containing $1 \mathrm{mg}$ of paranitrophenyl phosphate disodium (Sigma) in $1 \mathrm{~mL}$ of carbonate $\mathrm{MgCl}_{2}$ buffer ( $\mathrm{pH} 9.8$ ). The reaction was stopped with $2 \mathrm{~N} \mathrm{NaOH}$, and absorbance was measured at $405 \mathrm{~nm}$ with a photometer (Multiscan MCC/340, Labsystems, Helsinki, Finland). The results were expressed as EIA units (EIU) by multiplying the optical densities by 1,000 .

\section{Chlamydia pneumoniae serology}

C. pneumoniae IgA and IgG antibodies were measured previously [7] by microimmunofluorescence method (MIF) using the elementary bodies (EB) of Kajaani 6 (K6) strain of $C$. pneumoniae as an antigen, as described in detail elsewhere [8].

\section{Statistical analysis}

In this study, cut-off points for Hsp60 seropositivity were not determined, but the EIA units were divided into tertiles as follows: 1 st: $<123,2$ nd: $123-158$, and 3rd: $>158$ for $C$. pneumoniae Hsp60 IgA, and 1st: $<209$, 2nd: $209-252$, and 3rd: $>252$ for human Hsp60 IgA. Chi-squared test was then used to analyse the associations between cases and controls. The correlations of antibodies to C. pneumoniae Hsp60, human Hsp60, and $C$. pneumoniae K6 EB were determined using the Pearson correlation coefficient after $\log$ transformation of EIA units. After log transformation of EIA units, associations between Hsp antibodies and asthma status were tested using analysis of variance with age, sex and (ever-) smoking as covariates. In some models, C. pneumoniae IgA MIF seropositivity (0/1 variable with titre $1: 10$ as positive) was used as an additional covariate, and the two control groups combined due to small number of subjects. A p-value $<0.05$ was considered statistically significant.

\section{Results}

\section{Chlamydia pneumoniae antibodies}

The results of MIF showed that asthma patients had $C$. pneumoniae $\operatorname{IgG}$ antibodies present in their sera (titre $\geqslant 1: 16$ ) slightly more frequently compared with both control groups, seropositivity being $92 \%$ for asthma patients, $84 \%$ for asymptomatic controls and $88 \%$ for bronchitis controls. The frequency of $\operatorname{IgA}$ antibodies to $C$. pneumoniae (titre $\geqslant 1: 10$ ) was significantly higher in asthmatic patients compared only with asymptomatic controls $(\mathrm{p}<0.05)$, while acute bronchitis controls had even more IgA antibodies than cases: seropositivity was $72 \%$ for asthma patients, $44 \%$ for asymptomatic controls and $88 \%$ for bronchitis controls.

\section{Heat shock protein 60 antibodies}

When the antibodies against $C$. pneumoniae- and human-specific Hsp60 proteins were divided into 

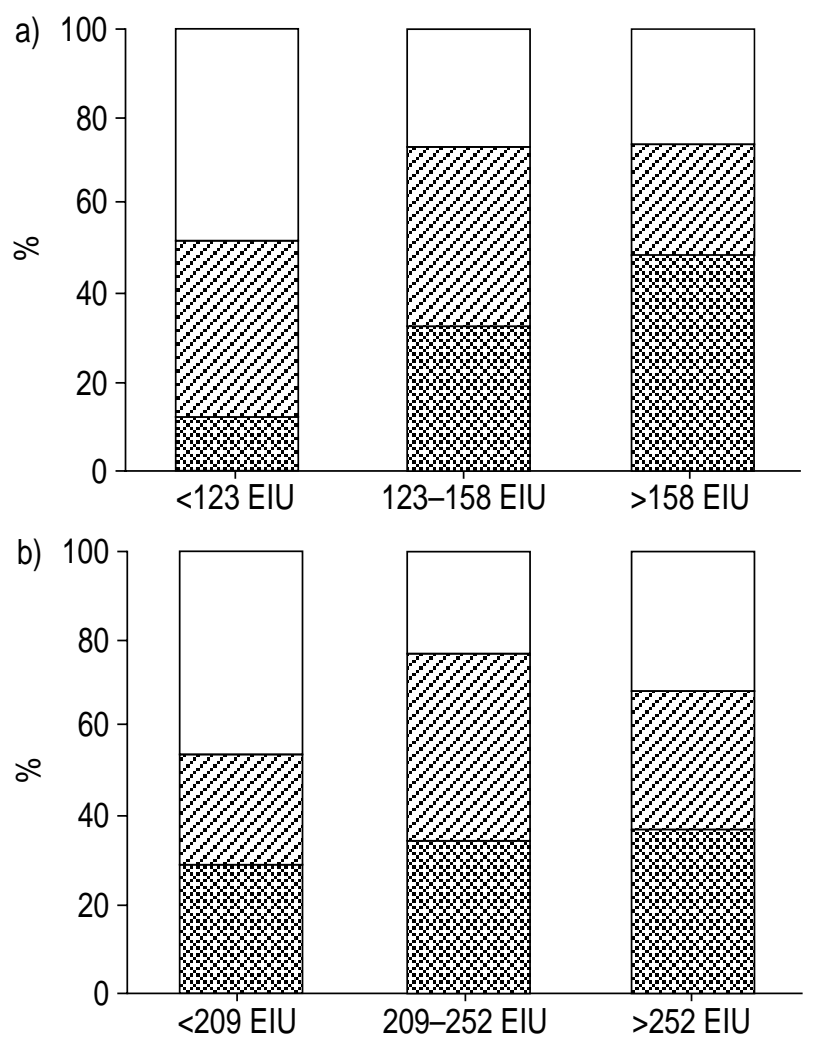

Fig. 1. - Percentage distribution of asthmatic subjects (网), asymptomatic controls $(\square)$ and bronchitis controls $(\mathbb{Z})$ in each tertile of immunoglobulin-(Ig)A antibodies measured by enzyme immunoassay (EIA) against the heat shock protein 60 of a) Chlamydia pneumoniae and b) human, presented as EIA units (EIU) (optical density multiplied by 1,000$)$.

tertiles, IgA antibodies against $C$. pneumoniae Hsp60 were strongly associated with asthma (fig. 1a), $p=0.12$ for asthma patients versus bronchitis controls and $\mathrm{p}=0.02$ for asthma patients versus both control groups. Controls with acute bronchitis did not differ significantly from asymptomatic controls $(\mathrm{p}=0.65)$. A strong $(\mathrm{r}=0.50)$ and significant $(\mathrm{p}<0.001)$ correlation was found between $C$. pneumoniae Hsp60 IgA antibodies and human Hsp60 IgA antibodies (fig. 2). The correlation was similar $(\mathrm{r}=0.49, \mathrm{p}<0.001)$ when only subjects demonstrating immune response to C. pneumoniae (IgG MIF titre $\geqslant 1: 32$ ) were analysed. Despite this correlation, no association was found between human Hsp60 IgA antibodies and asthma (fig. 1b). There was no significant correlation for human Hsp60 IgG antibodies and $C$. pneumoniae Hsp60 IgG antibodies.

The association remained between $C$. pneumoniae Hsp60 IgA antibodies and asthma in a comparison of geometric means adjusted for age, sex, and (ever-) smoking (table 2). The association using all controls stayed significant $(\mathrm{p}=0.02)$ even after $C$. pneumoniae IgA MIF seropositivity was added as an additional covariate. A similar trend was also seen in C. pneumoniae Hsp60 IgG antibodies, but the differences did not reach statistical significance. Only a weak correlation emerged between EIA IgA antibodies to C. pneumoniae Hsp60 and MIF IgA antibodies to

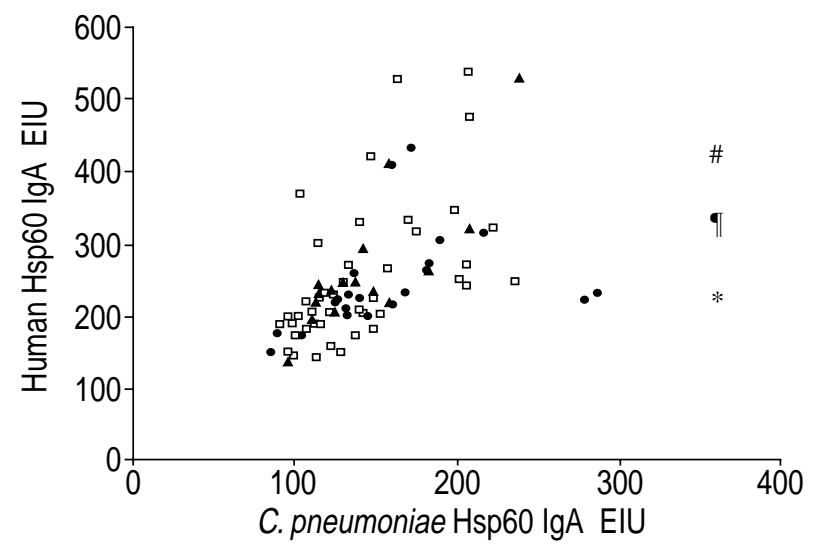

Fig. 2. - Comparison of immunoglobulin-(Ig)A antibodies measured by enzyme immunoassay (EIA) against the heat shock protein 60 (Hsp60) of Chlamydia pneumoniae and the Hsp60 of human in asthmatic subjects (○), asymptomatic controls $(\square)$ and bronchitis controls ( $\boldsymbol{\Delta})$, presented as EIA units (EIU) (optical density multiplied by 1,000$)$. *: asymptomatic control 638 EIU; ${ }^{*}$ : asthmatic subject 1,243 EIU; ${ }^{\circ}$ : asthmatic subject 810 EIU.

C. pneumoniae K6 EB $(\mathrm{r}=0.25, \mathrm{p}=0.02)$, whereas the corresponding $\mathrm{IgG}$ antibodies did not correlate with each other.

\section{Association with pulmonary function}

The C. pneumoniae Hsp60 IgA antibodies also inversely correlated with pulmonary function in the study group as a whole $(\mathrm{r}=-0.23, \mathrm{p}=0.04)$. When asthmatic patients were analysed separately, the correlation was similar $(\mathrm{r}=-0.21)$ but was not statistically significant.

\section{Discussion}

The presented results showing an association of C. pneumoniae Hsp60 IgA antibodies with asthma, support the hypothesis of an association between

Table 2.-Geometric means of immunoglobulin-(Ig)G and IgA antibodies measured by enzyme immunoassay (EIA) against the heat shock protein (Hsp) 60 of Chlamydia pneumoniae and human in asthmatic subjects, asymptomatic controls and bronchitis controls

\begin{tabular}{|c|c|c|c|}
\hline & $\begin{array}{l}\text { Asthmatic } \\
\text { subjects }\end{array}$ & $\begin{array}{l}\text { Asymptomatic } \\
\text { controls }\end{array}$ & $\begin{array}{l}\text { Bronchitis } \\
\text { controls }\end{array}$ \\
\hline Subjects $n$ & 24 & 45 & 17 \\
\hline \multicolumn{4}{|c|}{ C. pneumoniae Hsp60 EIU } \\
\hline $\mathrm{IgG}$ & 689 & 531 & 483 \\
\hline $\operatorname{IgA}$ & $184^{*}$ & 139 & 143 \\
\hline \multicolumn{4}{|c|}{ Human Hsp60, EIU } \\
\hline $\mathrm{IgG}$ & 399 & 392 & 443 \\
\hline $\operatorname{Ig} A$ & 244 & 234 & 251 \\
\hline
\end{tabular}

EIU: enzyme immunoassay unit; optical density multiplied by 1,$000 ; *: p=0.03$ versus asymptomatic controls, $p=0.01$ versus all controls (asymptomatic and bronchitis) using analysis of variance after log transformation of EIA units with age, sex and (ever-) smoking as covariates. 
C. pneumoniae infection and asthma [9]. Compared to asymptomatic controls, MIF IgA antibodies against $C$. pneumoniae were associated with both asthma and acute bronchitis, whereas EIA IgA antibodies to $C$. pneumoniae Hsp60 were associated only with asthma. No significant association was found between $\operatorname{IgG}$ antibodies and asthma, either in MIF antibodies against $C$. pneumoniae or in EIA antibodies to $C$. pneumoniae $\mathrm{Hsp} 60$, although the trend was obvious for Hsp60 antibodies. The lack of association between $\operatorname{IgG}$ antibodies to the same recombinant $C$. pneumoniae $\mathrm{Hsp} 60$ protein and asthma was also observed in another study in Finland, although antibodies were more common in cases than in controls even in that study (L. von Hertzen, The Finnish Lung Health Association, Helsinki, Finland; personal communication).

The association between C. pneumoniae Hsp60 IgA antibodies and asthma, and also the possible association of $C$. pneumoniae Hsp60 antibodies and the severity of pulmonary obstruction, support the possibility that the Hsp60 protein may play a role in the pathogenesis of bronchial hyperreactivity and/or pulmonary obstruction. Although the correlation between $C$. pneumoniae Hsp60 antibodies and pulmonary function was statistically significant, this finding requires confirmation in larger studies before it can be accepted with certainty. MiYAshita et al. [10] have shown marked anti-Hsp60 seroreactivity in an exacerbation of culture-proven persistent $C$. pneumoniae lung infection and suggested that an allergic reaction to Hsp could produce pulmonary symptoms.

The highly conserved nature of these proteins raises the possibility of molecular mimicry and generation of an autoimmune response as an additional factor in immunopathogenesis. HenRIKSEN et al. [11] have reported that in sera from normal blood donors there was no correlation between human Hsp60 IgG antibodies and $C$. pneumoniae $\mathrm{Hsp} 60 \mathrm{IgG}$ antibodies. No evidence was found for a significant correlation between human Hsp60 IgG antibodies and C. pneumoniae Hsp60 IgG antibodies. However, a significant correlation between the respective Hsp60 IgA antibodies was found. This correlation may be consistent with a role for Hsp60 molecular mimicry in respiratory illnesses. Conversely, the correlation could indicate that cross-reactive Hsp60 epitopes exist generally for IgA antibodies. Identification of specific reactive peptide sequences on $C$. pneumoniae and human Hsp60 would help to clarify these possibilities.

Asthma is associated with predominant Th2 and a relatively deficient Th1 phenotype. A similar Th2/ Th1 profile has been demonstrated in subjects with severe trachomatous scarring. In these patients, C. trachomatis Hsp60 increased the numbers of peripheral blood mononuclear cells producing interleukin-4, whereas $C$. trachomatis Hsp60 stimulated increased secretion of interferon-gamma in controls [12]. No comparable studies are available for $C$. pneumoniae-associated diseases. It is known, however, that chlamydial Hsp60 localizes in human atheroma and regulates macrophage tumour necrosis factor- $\alpha$ and matrix metalloproteinase expression [13]. An imbalance in metalloproteinase expression has also recently been implicated in the pathogenesis of airflow obstruction in asthma and chronic bronchitis [14].

MAYR et al. [15] recently found that serum antibodies against Hsp65/60 from subjects with atherosclerosis cross-react with human and chlamydial Hsp60, correlate with seropositivity against non-Hsp antigens of $C$. pneumoniae and mediate endothelial cytotoxicity. On this basis, they proposed that immune reactions to Hsp might mediate a possible link between infection and atherosclerosis. The present data correlating human and $C$. pneumoniae Hsp60 and MIF antibodies against $C$. pneumoniae, suggest a comparable role for immune reactions to Hsp in asthma.

It may also be possible that the Hsp60 responses simply represent a marker for exposure to chlamydial infection. Conversely, there are two counter-arguments in favour of a role for Hsp60 protein in the pathogenesis. Firstly, despite the weak but significant correlation between IgA antibodies to C. pneumoniae antigens in the MIF test and to C. pneumoniae Hsp60, the results indicate that there is, nevertheless, an independent association between $C$. pneumoniae Hsp60 IgA antibodies and asthma, even after controlling for the effects of MIF IgA antibodies to C. pneumoniae. A second argument is the differential IgA antibody responses in controls with acute bronchitis.

It is therefore felt that these findings support the need for further investigations on the role of Chlamydia pneumoniae heat shock protein 60 in the pathogenesis of asthma.

\section{References}

1. Hahn DL. Intracellular pathogens and their role in asthma: Chlamydia pneumoniae in adult patients. Eur Respir Rev 1996; 6: 224-230.

2. Peeling RW, Bailey RL, Conway DJ, et al. Antibody response to the $60-\mathrm{kDa}$ chlamydial heat-shock protein is associated with scarring trachoma. J Infect Dis 1998; 177: $256-259$.

3. Peeling RW, Kimani J, Plummer F, et al. Antibody to chlamydial hsp60 predicts an increased risk for chlamydial pelvic inflammatory disease. $J$ Infect Dis 1997; 175: $1153-1158$.

4. Toye B, Laferrière C, Claman P, Jessamine P, Peeling $\mathrm{R}$. Association between antibody to the chlamydial heat-shock protein and tubal infertility. $J$ Infect Dis 1993; 168: 1236-1240.

5. Brunham RC, Peeling R, Maclean I, Kosseim ML, Paraskevas M. Chlamydia trachomatis-associated ectopic pregnancy: serologic and histologic correlates. J Infect Dis 1992; 165: 1076-1081.

6. Beatty WL, Byrne G, Morrison RP. Repeated and persistent infection with Chlamydia and the development of chronic inflammation and disease. Trends in Microbiol 1994; 2: 94-98.

7. Hahn DL, Anttila T, Saikku P. Association of Chlamydia pneumoniae IgA antibodies with recently symptomatic asthma. Epidemiol Infect 1996; 117: $513-517$.

8. Wang S-P, Grayston JT. Population of prevalence antibody to Chlamydia pneumoniae, strain TWAR. 
In: Bowie WR, Caldwell HD, Jones RP, et al. eds. Chlamydial infections. Cambridge, Cambridge University Press, 1990; pp. $402-405$.

9. Hahn DL. Chlamydia pneumoniae, asthma and COPD: what is the evidence? Ann Allergy Asthma Immunol 1999; 83: 271-289.

10. Miyashita N, Matsumoto A, Kubota Y, Nakajima M, Niki Y, Matsushima T. Continuous isolation and characterization of Chlamydia pneumoniae from a patient with diffuse panbronchiolitis. Microbiol Immunol 1996; 40: $547-552$.

11. Henriksen T, Christiansen G, Birkelund S. Does infection with Chlamydia pneumoniae generate antibodies to $C$. pneumoniae GroEL and human HSP60? In: Saikku P, ed. Proceedings of the Fourth Meeting of the European Society for Chlamydia Research; 2000 Aug 20-23; Helsinki, Finland. Bologna, Societa Editrice Esculapio, 2000; p. 124.

12. Holland MJ, Bailey RL, Conway DJ, et al. T helper type-1 (Th1)/Th2 profiles of peripheral blood mononuclear cells (PBMC); responses to antigens of Chlamydia trachomatis in subjects with severe trachomatous scarring. Clin Exp Immunol 1996; 105: 429435.

13. Kol A, Sukhova GK, Lichtman AH, Libby P. Chlamydial heat shock protein 60 localizes in human atheroma and regulates macrophage tumor necrosis factor-alpha and matrix metalloproteinase expression. Circ 1998; 98: 300-307.

14. Vignola AM, Riccobono L, Mirabella A, et al. Sputum metalloproteinase-9/tissue inhibitor of metalloproteinase-1 ratio correlates with airflow obstruction in asthma and chronic bronchitis. Am J Respir Crit Care Med 1998; 158: 1945-1950.

15. Mayr M, Metzler B, Kiechl S, et al. Endothelial cytotoxicity mediated by serum antibodies to heat shock proteins of Escherichia coli and Chlamydia pneumoniae. Immune reactions to heat shock proteins as a possible link between infection and atherosclerosis. Circ 1999; 99: 1560-1566. 